\title{
Evidence for a long time-course adaptation of glucose metabolism to high-protein feeding in rats without major changes in energy expenditure
}

\author{
M. Stepien, D. Azzout-Marniche, P. Even, A. Payet, A. Simonin, G. Fromentin, D. Tome \\ and C. Gaudichon \\ UMR 914, INRA-AgroParisTech, Paris, France
}

\begin{abstract}
High-protein diets are known to participate in weight reduction by lowering spontaneous energy intake ${ }^{(1)}$. An increase in the thermic effect of feeding is thought to be responsible for this effect ${ }^{(2)}$. However, little is known about the changes in energy expenditure that occur during adaptation and especially in relation to nutrient oxidation in the fasted and the fed state. The aim of the present study was to characterize these changes.

Male Wistar rats $(n$ 44) weighing $200 \mathrm{~g}$ and equipped with a permanent vena cava catheter were fed a normal-protein diet (NP) for 1 week and then a high-protein diet (HP). Energy metabolism was measured in an open-circuit indirect calorimetry after $1,3,6$ or $15 \mathrm{~d}$ of adaptation. Animals received a continuous saline $(9 \mathrm{~g} \mathrm{NaCl} / \mathrm{l})$ perfusion to increase urine volume. They were fasting overnight and then received a meal of $4 \mathrm{~g}$. The postprandial metabolism was followed for $4 \mathrm{~h}$. At the end of this period the rats were killed.

During the fasting state the total and resting energy expenditure (REE) were similar among groups. REE ranged from 1.9 (SD 0.3 ) W in the HP rats after $1 \mathrm{~d}$ to 2.03 (SD 0.2) W in the NP group. Compared with NP rats the thermic effect of feeding was only transiently increased after $6 \mathrm{~d}$ on the HP diet $(5.6(\mathrm{SD} 2.4) \mathrm{kJ} v .4 .1$ (SD 1.9) kJ in the NP group). During the fasting state there was a trend to decreased fat oxidation after $3 \mathrm{~d}$ on the HP diet at the expense of protein oxidation. In the fed state fat oxidation greatly increased after $1 \mathrm{~d}$ on the of $\mathrm{HP}$ diet (from 2.8 (SD 3.7) kJ in NP rats to 9.7 (SD 3.1) kJ) but then decreased after $3 \mathrm{~d}$ and remained stable until $15 \mathrm{~d}(3.7$ (SD 3.3 ) kJ). Interestingly, carbohydrate oxidation was twofold lower after $1 \mathrm{~d}$ on the HP diet and then progressively increased until $15 \mathrm{~d}$ but remained lower than that for NP rats $(25.3$ (SD 5.9) kJ for NP group $v .19 .8$ (SD 1.7) kJ for HP group after $15 \mathrm{~d} ; P<0.05$ ).

The present study shows that the thermic effect of feeding is the unique energy expenditure component that is increased after an HP diet, but only transiently. Feeding an HP diet resulted in dramatic changes in postprandial nutrient oxidation that particularly suggests a slow adaptation of carbohydrate metabolism. The activation of either gluconeogenesis or glycogen turnover progressively takes place from $3 \mathrm{~d}$ to $15 \mathrm{~d}$ of feeding. A concomitant tracer study is underway to investigate specifically the transfer of dietary amino acids in energy pathways.
\end{abstract}

1. Blouet C, Mariotti F, Azzout-Marniche D, Bos C, Mathe V, Tome D \& Huneau JF (2006) J Nutr 136, 1849-1854.

2. Petzke KJ, Riese C \& Klaus S (2007) J Nutr Biochem 18, 400-407. 